\title{
PfSETvs methylation of histone H3K36 represses virulence genes in Plasmodium falciparum
}

\author{
Lubin Jiang ${ }^{1 *}$, Jianbing $\mathrm{Mu}^{2 *}$, Qingfeng Zhang ${ }^{3,4,5}$, Ting $\mathrm{Ni}^{6}$, Prakash Srinivasan ${ }^{2}$, Kempaiah Rayavara ${ }^{2}$, Wenjing Yang ${ }^{7}$, \\ Louise Turner $^{8}$, Thomas Lavstsen ${ }^{8}$, Thor G. Theander ${ }^{8}$, Weiqun Peng ${ }^{9}$, Guiying Wei ${ }^{3}$, Qingqing Jing ${ }^{1}$, Yoshiyuki Wakabayashi ${ }^{7}$, \\ Abhisheka Bansal ${ }^{2}$, Yan Luo ${ }^{7}$, José M. C. Ribeiro ${ }^{2}$, Artur Scherf ${ }^{4,5}$, L. Aravind ${ }^{10}$, Jun Zhu ${ }^{7}$, Keji Zhao ${ }^{11}$ \& Louis H. Miller ${ }^{2}$
}

The variant antigen Plasmodium falciparum erythrocyte membrane protein 1 (PfEMP1), which is expressed on the surface of $P$. falciparum-infected red blood cells, is a critical virulence factor for malaria ${ }^{1}$. Each parasite has $\mathbf{6 0}$ antigenically distinct var genes that each code for a different PfEMP1 protein. During infection the clonal parasite population expresses only one gene at a time before switching to the expression of a new variant antigen as an immuneevasion mechanism to avoid the host antibody response $\mathrm{e}^{2,3}$. The mechanism by which 59 of the 60 var genes are silenced remains largely unknown ${ }^{4-7}$. Here we show that knocking out the $P$. falciparum variant-silencing $S E T$ gene (here termed PfSETvs), which encodes an orthologue of Drosophila melanogaster ASH1 and controls histone $\mathrm{H} 3$ lysine 36 trimethylation (H3K36me3) on var genes, results in the transcription of virtually all var genes in the single parasite nuclei and their expression as proteins on the surface of individual infected red blood cells. PfSETvs-dependent $\mathrm{H} 3 \mathrm{~K} 36 \mathrm{me} 3$ is present along the entire gene body, including the transcription start site, to silence var genes. With low occupancy of PfSETvs at both the transcription start site of var genes and the intronic promoter, expression of var genes coincides with transcription of their corresponding antisense long noncoding RNA. These results uncover a previously unknown role of PfSETvsdependent $\mathrm{H} 3 \mathrm{~K} 36 \mathrm{me} 3$ in silencing var genes in $P$. falciparum that might provide a general mechanism by which orthologues of PfSETvs repress gene expression in other eukaryotes. PfSETvs knockout parasites expressing all PfEMP1 proteins may also be applied to the development of a malaria vaccine.

In addition to histone deacetylases (HDACs) ${ }^{8,9}$, histone lysine methyltransferases (HKMTs) or histone lysine demethylases (HKDMs) may have critical roles in controlling gene expression in $P$. falciparum $^{4-7,10,11}$. There are a total of ten predicted $P$. falciparum HKMTs (PfHKMTs) belonging to the SET domain superfamily, two PfHKDMs of the LSD1 family and three PfHKDMs of the Jumonji-related family ${ }^{10,12}$ (Supplementary Table 1). However, the key factor for var gene silencing remains unknown.

We therefore examined whether PfHKMTs or PfHKDMs are key factors in controlling mutually exclusive expression of the var gene family by attempting to knock out all of the PfHKMT (PfSET) genes and three of the PfHKDM genes in a P. falciparum clone, 3D7 (Fig. 1a and Supplementary Fig. 1). Four out of nine PfSET genes and all three studied PfHKDM genes could be genetically disrupted (Fig. $1 \mathrm{~b}$ and Supplementary Fig. 1), suggesting that the other five PfSET genes are essential for the parasite in the asexual blood stage. Gene expression microarray analyses showed that the knockout (Fig. 1c, d and Supplementary Fig. 1c) of the gene previously referred to as PfSET2 (ref. 10) (PlasmoDB gene ID: PF3D7_1322100) led to the expression of virtually all var genes in the ring stage (Fig. 1e and Supplementary Table 2). By contrast, knockout of any other PfSET or PfHKDM genes did not alter the transcription of the var gene family in 3D7 (Supplementary Fig. 1e-j and Supplementary Tables 3-8). In addition, some members of other clonally variant gene families (rifin and stevor) plus the var gene family account for most of the genes upregulated in the P. falciparum 3D7 lacking the SET2 gene (3D7SET2 $\Delta$ ) (Supplementary Fig. 2 and Supplementary Table 2). Therefore, we renamed this $P$. falciparum variant-silencing SET gene PfSETvs. Activation of the majority of var genes by SETvs $\Delta$ was further corroborated by quantitative PCR (qPCR) at $18 \mathrm{~h}$ after invasion in both 3D7 (Fig. 1f) and another P. falciparum clone, Dd2 (Supplementary Fig. 3), indicating that PfSETvs is involved in broadly silencing var genes.

To determine whether PfSETvs $\Delta$ activated multiple var genes in a single infected red blood cell (iRBC), we tested whether different types of var genes could be transcribed in a single 3D7SETvs $\Delta$ iRBC by RNA fluorescence in situ hybridization (FISH). Each combined RNA FISH of two representative var transcripts indicated co-expression of all three types of var genes in an individual 3D7SETvs $\Delta$ nucleus (Fig. 2a). The tested var transcripts colocalized with each other at a particular site of the nuclear periphery (Fig. 2a). Transcription of a control gene, seryltRNA synthetase (PF3D7_0717700), did not occur at this site (Fig. 2a), suggesting that var genes have a specific transcriptionally active site, in agreement with previous findings ${ }^{6,13}$. Moreover, our results showed that multiple var transcripts also colocalized at the single peripheral site of 3 D7SETvs $\Delta$ nuclei, even though the genomic loci of these var genes were diverse (Supplementary Fig. $4 \mathrm{a}-\mathrm{c}$ ). Taken together, our results demonstrate multiple var transcripts in one nucleus and suggest that a var-specific nuclear compartment exists for active transcription of multiple var genes.

To determine whether parasites transcribing multiple var genes are able to translate and transport multiple PfEMP1 proteins to the surface of iRBCs, a live-cell immunofluorescence assay (IFA) was performed with rat and rabbit antibodies to different PfEMP1 proteins. As expected, the gelatin-enriched parasite presented knobs on the surface of iRBCs in both 3D7 and 3D7SETvs $\Delta$ (Fig. 2b, c). Furthermore, surface expression of multiple PfEMP1 proteins on a single $3 \mathrm{D} 7$ SETv $\triangle \mathrm{iRBC}$ was observed by confocal microscopy (Fig. $2 \mathrm{~d}$ and Supplementary Fig. 4d). It is

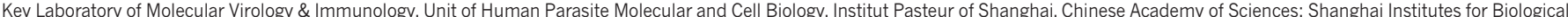

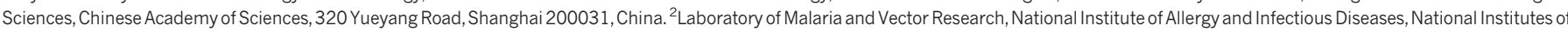

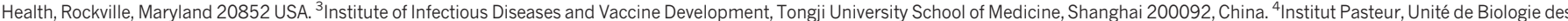
Interactions Hôte-Parasite, Département de Parasitologie et Mycologie, F-75015 Paris, France. ${ }^{5}$ CNRS, URA 2581, F-75015 Paris, France. ${ }^{6}$ State Key Laboratory of Genetics Engineering \& MOE Key

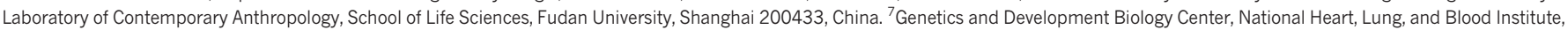

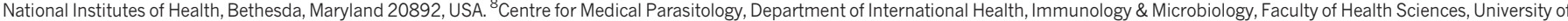

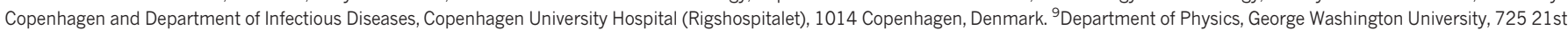

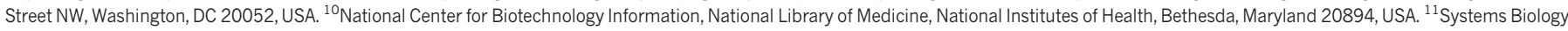
Center, National Heart, Lung, and Blood Institute, National Institutes of Health, Bethesda, Maryland 20892, USA.
}

*These authors contributed equally to this work. 
a

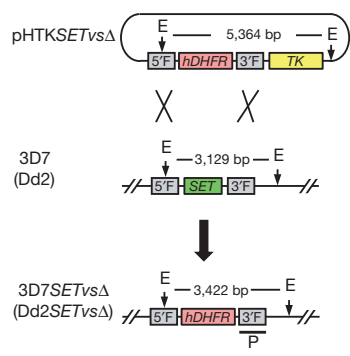

$\mathbf{f}$

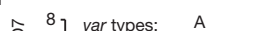

b

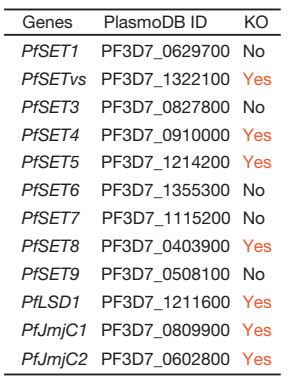

c

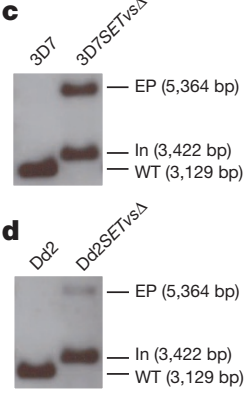

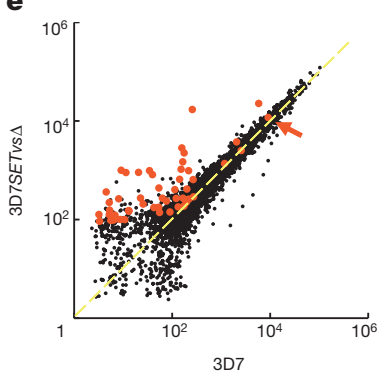

C

B E

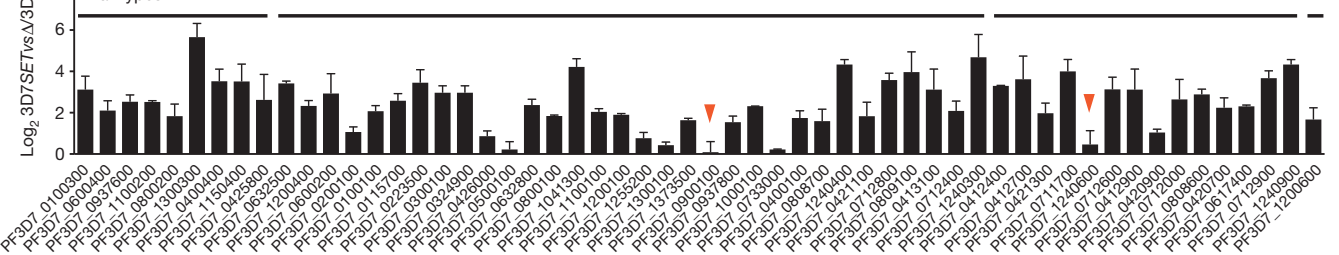

Figure $1 \mid$ Knockout of PfSETvs leads to expression of all var genes. a, Schematic diagram of the PfSETvs gene knockout strategy by using plasmid pHTK. 3'F, 3' flanking fragment for crossover recombination; 5' F, 5' flanking fragment for crossover recombination; E, EcoRV; $h D H F R$, human dihydrofolate reductase; P, DNA probe for Southern blot analysis; TK, thymidine kinase; SET, SET domain. $\mathbf{b}$, Summary of knockout studies for nine PfHKMTs and three PfHKDMs (PfLSD1, PfJmjC1 and PfJmjC2) genes. KO, knockout; no, failed to knockout gene; yes, succeeded in gene knockout. c, d, Southern blot analysis using a DNA probe (P) from downstream of the knocked out SET domain of the PfSETvs gene (see also panel a) for PfSETvs $\triangle$ in 3D7 (c) and Dd2 (d). The sizes of three different hybridization bands from the integrated (In) or wild-type (WT) genomes and the episomal plasmid (EP) are

important to note that in 3D7SETvs $\triangle$ iRBCs double labelling of PfEMP1 proteins was always observed (Fig. 2d). As reported previously ${ }^{14}$, no coexpression of different PfEMP1 proteins in individual iRBCs by $3 \mathrm{D} 7$ clones was detected using different antibodies (Fig. 2d and Supplementary Fig. 4 d). We were also unable to show surface labelling of the active PF3D7_1240600 in the wild-type 3D7 because we lacked an antibody to this PfEMP1.

PfSETvs, an orthologue of D. melanogaster ASH1, is the only representative of the SETD2-NSD-ASH1 clade in P. falciparum (Supplementary indicated to the right. bp, base pairs. e, Comparative transcriptome analysis of wild-type 3D7 and 3D7SETvs $\Delta$ at $18 \mathrm{~h}$ after invasion. $x$ axis (wild-type 3D7) and $y$ axis (3D7SETvs $\Delta$ ) are logarithmic and correspond to relative signal of hybridization to each gene shown as a dot (see also Supplementary Table 2). All var genes with authentic hybridization signals are shown in red. The dominantly expressed var gene (PF3D7_1240600) in wild-type 3D7 is indicated by a red arrow. f, qPCR analyses of transcriptional upregulation $\left(\log _{2}\right.$ ratio of PfSETvs $\Delta$ to wild-type parasites) of var genes in $3 D 7 S E T v s \Delta$ at $18 \mathrm{~h}$ after invasion. Type of var gene (A, B, C or E) is shown at the top. The dominantly expressed var gene and a second gene expressed at a low frequency in the wild-type 3D7 population are indicated by red arrowheads. Experiments were repeated three times. Error bars represent s.e.m.

Fig. 5), which, in addition to the SMYD clade, are the two distinct occasions in the evolution of SET domains as H3K36-specific methyltransferases in eukaryotes ${ }^{12}$. To monitor changes of histone lysine methylations by PfSETvs $\Delta$, antibodies that specifically recognized P. falciparum H3K36me3, H3K36me2 (Supplementary Fig. 6a, b), $\mathrm{H} 3 \mathrm{~K} 4 \mathrm{me} 3, \mathrm{H} 3 \mathrm{~K} 9 \mathrm{me} 3$ and H4K20me3 were used in chromatin immunoprecipitation combined with massively parallel DNA sequencing (ChIP-seq) experiments. In the wild-type 3D7, a robust enrichment of H3K36me3 (Fig. 3a-c) but not H3K36me2 (Supplementary Fig. 7a)
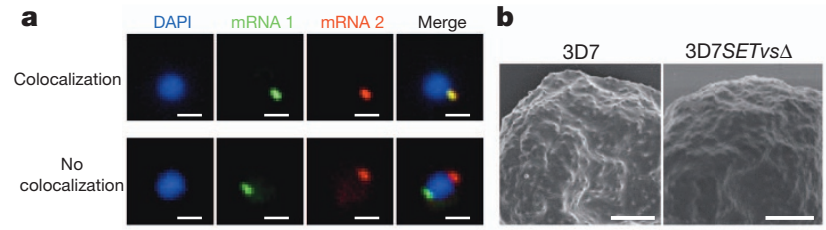

c
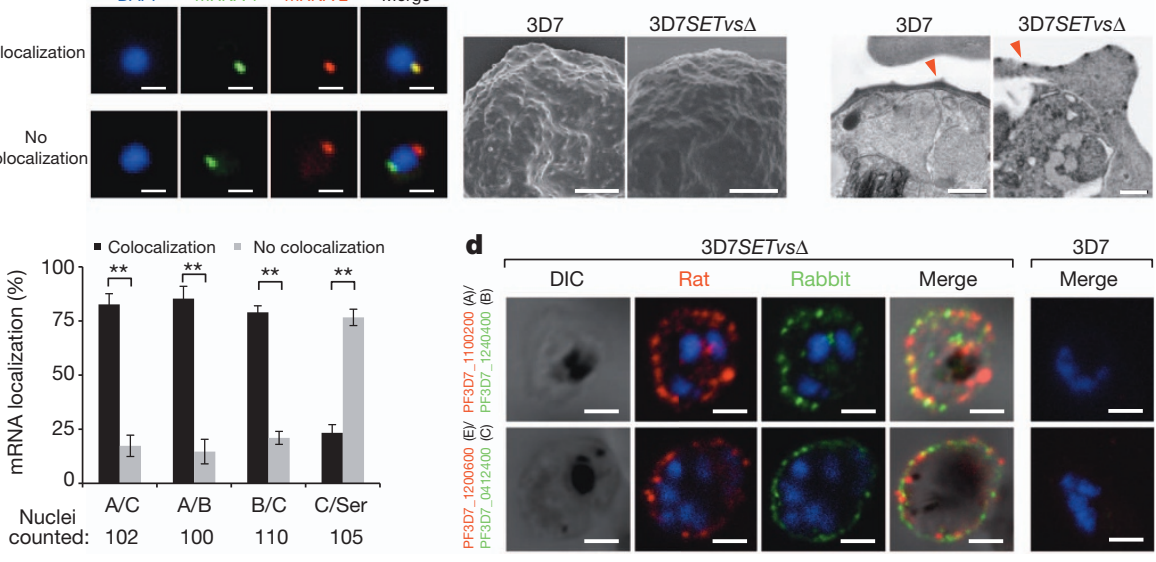

Figure 2 Simultaneous expression of multiple var genes in single 3D7SETvs $\Delta$ iRBCs. a, Two-colour RNA FISH (top) and statistical analyses of colocalization (bottom) of each two types of var transcripts in 3D7SETvs $\Delta$ by using gene-specific probes (Supplementary Fig. 4a). Seryl-tRNA synthetase (Ser) transcript served as a negative control. Average numbers of counted nuclei are listed under each tested group. $n=3$. Error bars represent s.e.m. $P$ values were obtained using a one-tailed Student's $t$-test. $* * P<0.01$. b, c, Electron microscopy of gelatin-selected 3D7 and 3D7SETvs $\triangle$ iRBCs.
Typical knobs in scanning electron microscopy (b) and transmission electron microscopy (c) pictures are indicted by red arrowheads. d, Live-cell IFA using rat and rabbit antisera to various PfEMP1 proteins to detect co-expression of different PfEMP1 proteins on the surface of 3D7SETvs $\triangle$ iRBCs. Wild-type 3D7 $\mathrm{iRBC}$ is shown to the right. No staining is seen. DAPI ( $4^{\prime}, 6$-diamidino-2phenylindole, blue) is used to mark the parasite nucleus. Types of var genes are shown in parentheses. Scale bars, $1 \mu \mathrm{m}(\mathbf{a}, \mathbf{b}), 0.5 \mu \mathrm{m}$ (c) and $1.5 \mu \mathrm{m}$ (d). 
was observed only in the telomeric and subtelomeric heterochromatin regions of the $14 \mathrm{P}$. falciparum chromosomes plus several discrete genomic regions where all of the var genes are located at either 18 or $42 \mathrm{~h}$ after invasion. However, compared with other histone lysine methylations, $\mathrm{H} 3 \mathrm{~K} 36 \mathrm{me} 3$ was greatly reduced in the entire gene body of var genes in 3D7SETvs $\Delta$ at $18 \mathrm{~h}$ after invasion (Fig. 3d and Supplementary Figs 8 and 9), indicating a direct positive correlation of H3K36me3 with PfSETvs activity. Considering the extremely low level of H3K36me2 at var loci in wild-type 3D7 (Supplementary Fig. 7a), only $\mathrm{H} 3 \mathrm{~K} 36 \mathrm{me} 3$ is functionally important for var gene regulation. PfSETvs may di- and trimethylate $\mathrm{H} 3 \mathrm{~K} 36$, as these markers were also reduced at the transcription start site (TSSs) of activated var genes owing to PfSETvs $\Delta$ (Supplementary Fig. 7c-g). Interestingly, similarly high levels of H3K36me3 were observed in both wild-type 3D7 and 3D7SETvs $\Delta$ at $42 \mathrm{~h}$ after invasion when var genes were silent (Fig. 3e), indicating at least one other PfHKMT that catalyses H3K36me3 in $P$. falciparum schizont iRBCs. In addition, our data showed that none of the var transcripts colocalized with H3K36me3 in the nuclei (Supplementary Fig. 10). Collectively, our data suggest that the PfSETvs-dependent H3K36me3 is specifically involved in var gene silencing.
Notably, H3K36me3 was also observed for a high enrichment at the $3^{\prime}$ end of 400 ring-stage-active genes (other than var, rifin and stevor genes) compared to 400 ring-stage-silent genes (see gene lists in Supplementary Table 9) in both wild-type 3D7 and 3D7SETvs $\Delta$ (Fig. 3f, g), indicating that PfSETvs-independent H3K36me3 may contribute to transcriptional elongation, as reported in other eukaryotes ${ }^{15-17}$, and might compensate for the global levels of H3K36me3 in 3D7SETvs $\triangle$ (Supplementary Fig. 6c, d). We next examined whether the reduction of H3K36me3 by PfSETvs $\Delta$ is specifically associated with activation of parasite clonally variant genes. Among 5,276 P. falciparum genes, 59 out of 59 var genes, 97 out of 150 rifin genes (including 69 A-type and 28 B-type rifin genes) and 18 out of 29 stevor genes belonged to the top 250 genes with highest reduction of H3K36me3 by PfSETvs $\Delta$ (Fig. 3h). Furthermore, the same gene group is enriched for increased expression as determined by microarray experiments (Supplementary Table 10). Our data indicate that $\mathrm{H} 3 \mathrm{~K} 36 \mathrm{me}$, controlled by PfSETvs, has a repressive role in silencing parasite clonally variant gene families.

To corroborate further the role of $\mathrm{H} 3 \mathrm{~K} 36 \mathrm{me} 3$ in var gene silencing, we examined histone modification at the TSS of an active var gene (PF3D7_1240600) and a silent var gene (PF3D7_1200600) in the wildtype 3D7, both of which are active in 3D7SETvs $\Delta$ (Fig. 4a, b). Because of

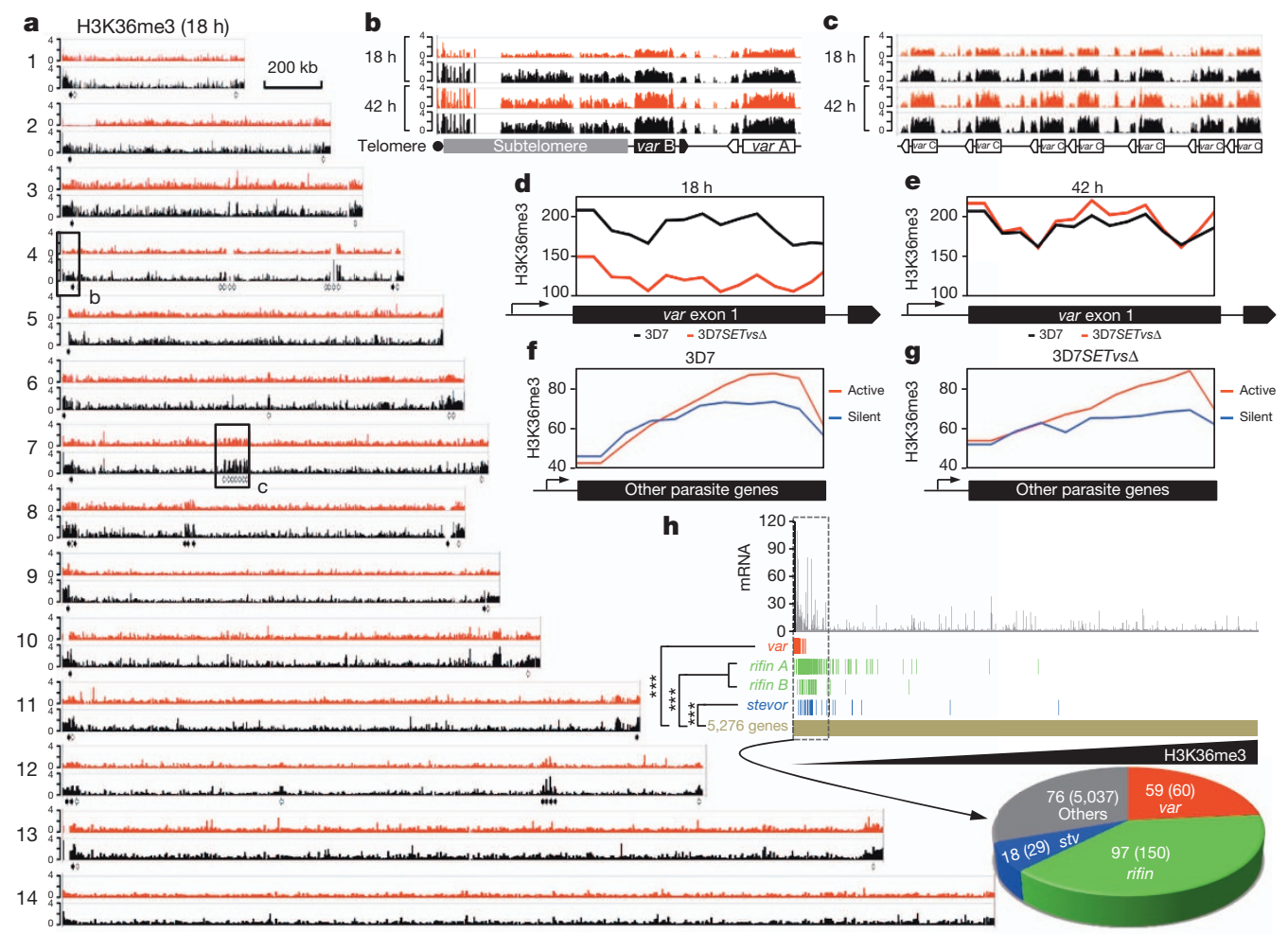

Figure 3 PfSETvs-dependent H3K36me3 is specifically associated with var gene silencing. a, Integrative genomic view of ChIP-seq analysis of $\mathrm{H} 3 \mathrm{~K} 36 \mathrm{me} 3$ along 3D7 (black) and 3D7SETvs $\Delta$ (red) chromosomes at $18 \mathrm{~h}$ after invasion. Sixty var genes distributed along $P$. falciparum chromosomes 1-13 are indicated by solid (forward orientation) and open (reverse orientation) arrows. Each read was normalized by the total number of uniquely mapped ChIP-seq reads. Chromosomal numbers are shown to the left. Regions are boxed for a detailed view represented in $\mathbf{b}$ and $\mathbf{c}$. A scale bar representing 200 kilobases $(\mathrm{kb})$ is shown to the right of chromosome 1 . b, c, At $18 \mathrm{~h}$ and $42 \mathrm{~h}$ after invasion, integrative genomic view of $\mathrm{H} 3 \mathrm{~K} 36 \mathrm{me} 3$ distributed at the $5^{\prime}$ end of chromosome 4 representing a region that includes the telomere, subtelomere, type A and B var genes (b), and at the middle of chromosome 7 representing a type C var gene cluster (c) in 3D7 (black) and 3D7SETvs $\Delta$ (red) d, e, Distribution of H3K36me3 along exon 1 of 50 tested var genes in $3 \mathrm{D} 7 \operatorname{SET} v s \Delta$ (red) and wild-type 3D7 (black) at $18 \mathrm{~h}(\mathbf{d})$ or $42 \mathrm{~h}$ (e) after invasion. Exon 1 of each var gene was equally divided into 14 bins. Total reads of each bin by ChIP-seq were normalized by total uniquely mapped reads. f, g, Distribution of H3K36me3 across the gene bodies of 400 ring-stage-active genes (red) and 400 ring-stage-silent genes (blue) (see gene list in Supplementary Table 9) in wild-type 3D7 (f) and 3D7SETvs $\Delta(\mathbf{g})$. Each gene was equally divided into 20 bins. Total reads of each bin by ChIP-seq were normalized by total uniquely mapped reads. $\mathbf{h}$, Statistical analysis of the correlation between reduction of $\mathrm{H} 3 \mathrm{~K} 36 \mathrm{me} 3$ and upregulation of var, rifin and stevor gene families. 5,276 parasite genes were sorted from low to high levels of H3K36me3 in 3D7SETvs $\Delta$ normalized by that in 3D7. Expression fold change of each gene by PfSETvs $\Delta$ was shown on the top panel (see also Supplementary Table 10). Distribution of all of $\operatorname{var}$ (red), rifin, including A- and B-type rifin genes (green) and stevor (blue) genes is shown along the parasite genes (gold). In the top $250 \mathrm{H} 3 \mathrm{~K} 36 \mathrm{me} 3$-reduced genes boxed by dash lines, numbers of var (red), rifin (green), stevor (stv, blue) and other genes (grey) compared to their total numbers were shown in a pie chart at the bottom. Hypergeometric test was computed for the $\operatorname{var}\left(P=3.4 \times 10^{-80}\right)$, rifin $\left(P=9.7 \times 10^{-98}\right)$ and stevor $\left(P=1.73 \times 10^{-17}\right)$ gene families to gauge their significance of upregulation in the reduction of $\mathrm{H} 3 \mathrm{~K} 36 \mathrm{me} 3$. 
high sequence similarity in the $5^{\prime}$-untranslated region, including the TSS and the intronic promoter of var genes, ChIP-qPCR but not ChIP-seq can be used in these regions (Fig. 3b, c). In wild-type 3D7, the TSS occupancy of H3K36me3 is considerably higher in the silent var gene compared to the active one (Fig. $4 \mathrm{a}, \mathrm{b}$ ). By contrast, the two var genes studied both exhibited low levels of H3K36me3 at the TSS in 3D7SETvs $\Delta$, consistent with their active expression (Fig. 4a, b). H3K9me3, a transcriptional silent mark, showed similar profiles as H3K36me3 (Fig. 4a, b), whereas two active marks, H3K4me3 and H4 acetylation, were present at the TSSs of active genes in both wild-type 3D7 and 3D7SETvs $\Delta$ (Fig. 4a, b). In addition, the similar results were observed in three other var genes representing type A (PF3D7_0400400), type B (PF3D7_0300100) and type C (PF3D7_0617400) (Supplementary Fig. 11). Altogether, our data support the idea that the high level of $\mathrm{H} 3 \mathrm{~K} 36 \mathrm{me} 3$ at the TSS region is involved in transcriptional repression.

It is worth noting that each var gene harbours an intronic promoter driving the transcription of an antisense long non-coding RNA (lncRNA) of unknown function ${ }^{18}$. Our ChIP-seq data showed that two active var genes (PF3D7_1240600 and PF3D7_0900100) in wildtype $3 \mathrm{D} 7$ populations (Fig. 1f) had low levels of $\mathrm{H} 3 \mathrm{~K} 36 \mathrm{me} 3$ at the $3^{\prime}$ end of exon 1, whereas silent var genes had high levels of H3K36me3 at the same region (Supplementary Fig. 12), suggesting a positive correlation between PfSETvs-dependent H3K36me3 occupancy and var lncRNA silencing. To explore this concept further, histone modification profiles in the $3^{\prime}$ portion of var exon 1 as a proxy for the lncRNA promoter was examined by ChIP-qPCR, as the introns of var genes are highly conserved among the gene family. Our results showed similar trends of H3K36me3 between the TSSs of var genes and their corresponding $3^{\prime}$ but not $5^{\prime}$ portions of exon 1 (Fig. $4 \mathrm{a}, \mathrm{b}$ and Supplementary Fig. 11), consistent with the observation by strand-specific qPCR that active transcription of var genes coincides with the expression of the corresponding antisense $\operatorname{lncRNAs}$ at 8-18 h after invasion (Fig. 4c, d and Supplementary Fig. 13). These results demonstrated a correlated upregulation of var genes and their corresponding lncRNAs in association with low occupancy of the PfSETvs-dependent H3K36me3 at the TSS.
To investigate further the biological function of PfSETvs in var gene silencing, a triple haemagglutinin (HA) tag was fused in frame to the carboxy terminus of PfSETvs in 3D7SETvsHA (Supplementary Fig. 14a-d). The resulting PfSETvs-HA protein, like wild-type PfSETvs, still contributed to the mutually exclusive expression of the var gene family (Supplementary Fig. 14e). Furthermore, IFA analysis showed that PfSETvs-HA located at multiple nuclear sites, one of which colocalized with H3K36me3 in 3D7SETvsHA (Supplementary Fig. 14f), suggesting that the enzymatic activity of PfSETvs for H3K36me3 might require additional factors at the single perinuclear site. ChIPqPCR results showed that, at $18 \mathrm{~h}$ after invasion, PfSETvs-HA was not enriched at the TSS and in the intronic promoter region of the active var gene (Fig. 4e), and instead tended to increase at these regions of silent var genes tested in 3D7SETvsHA (Fig. 4f and Supplementary Fig. 14g-i). No comparable enrichment of PfSETvs-HA was observed in a var-unrelated silent gene (PF3D7_0424100) (Supplementary Fig. 14j). Taken together, our data indicate that PfSETvs-HA specifically localizes to the TSSs and intronic promoters for var gene silencing, in association with the PfSETvs-dependent H3K36me3 (Fig. 4g).

In this study we have shown that the H3K36 methylation system is differentiated into at least two distinct forms in P. falciparum, with the PfSETvs-dependent system functioning in a negative regulatory capacity (Fig. 4g), and the second independently of it alongside the elongating RNAPII (Supplementary Fig. 14k). Cognates of the PfSETvs-dependent mechanism for gene silencing might also exist in other eukaryotes in the cases of previously reported members of the ASH1-like subclade, such as Caenorhabditis elegans MES-4 (ref. 19) and D. melanogaster ASH1 (ref. 20), and perhaps explain the association between H3K36me3 and silent genes in zebrafish sperm ${ }^{21}$ and the pericentromeric heterochromatin in mouse embryonic stem cells and fibroblasts ${ }^{22}$. In the RNAPIIrelated mechanism, H3K36me3 generated by the SETD2 subclade enzymes recruits $\mathrm{HDACs}^{15}$ and prevents incorporation of acetylated histones ${ }^{23}$ in transcribed gene bodies to prevent cryptic transcription initiation inside active genes. Given the role of $\operatorname{lncRNAs}$ as scaffolds a
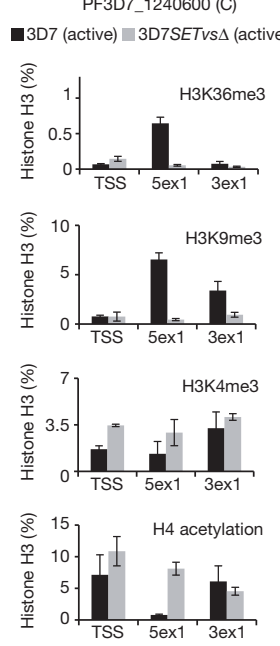

b
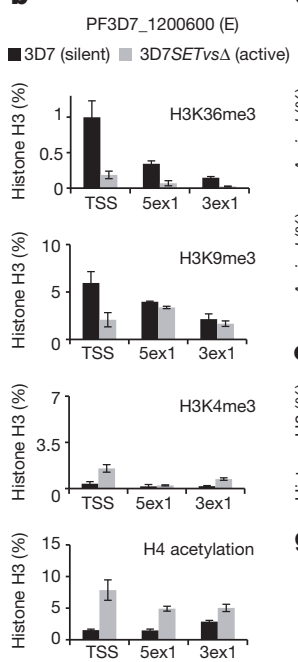

c

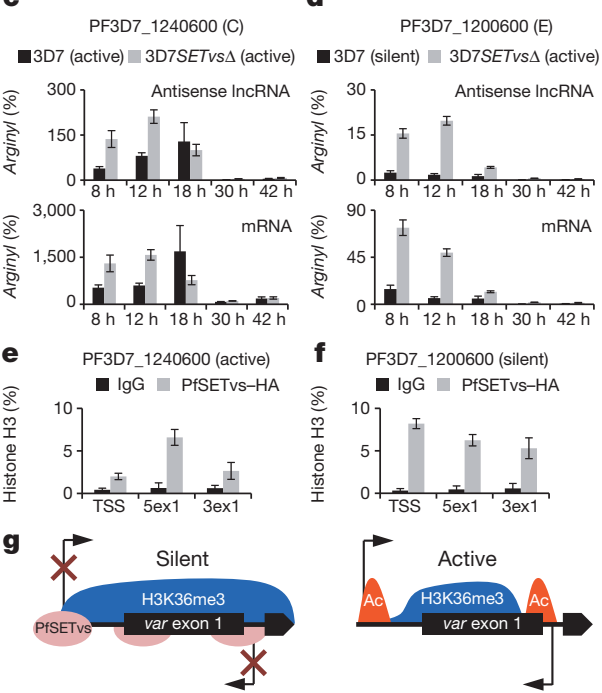

3ex1 PCR primer set (Supplementary Fig. 7b) were used for antisense lncRNA and mRNA reverse transcription, respectively. Type of var gene and its transcription status are shown in parentheses. Experiments were repeated three times. Error bars represent s.e.m. e, f, ChIP-qPCR of the active 3D7 var gene PF3D7_1240600 (e) and a silent 3D7 var gene PF3D7_1200600 (f) with a mouse antibody to HA in 3D7SETvs-HA at $18 \mathrm{~h}$ after invasion by using the same PCR primers in a. g, Summary diagram showing that the PfSETvsdependent H3K36me 3 enriched along the entire gene body of silent var genes, including the TSS of var genes and the respective intronic antisense promoter, leads to silencing of both var mRNA and antisense lncRNA. Ac, acetylation. time points after invasion as shown in the figures. Expression levels of var transcripts were normalized to expression of a housekeeping gene, arginyltRNA synthetase (PF3D7_0913900). The forward and reverse primers of the
Figure 4 PfSETvs and H3K36me3 repress var gene expression at the TSS. a, b, ChIP-qPCR of the active 3D7 var gene PF3D7_1240600 (a) and a silent H3K4me3 and histone H4K5/K8/K12/K16 acetylation in both 3D7 and 3D7SETvs $\Delta$ at $18 \mathrm{~h}$ after invasion by using three different PCR primer sets schematized in Supplementary Fig. 7b. 3ex1, 3' end of exon 1; 5ex1, 5' end of exon 1. c, d, Expression profiles of messenger RNA and antisense lncRNA transcribed from PF3D7_1240600 (c) or PF3D7_1200600 (d) at five different 226 | NATURE | VOL 499 | 11 JULY 2013 
recruiting Set2 histone methyltransferase and Set3 histone deacetylase complex to repress transcription initiation in yeast ${ }^{24,25}$, it would be interesting to investigate whether the antisense lncRNA might regulate var gene expression in a similar manner ${ }^{25}$.

The factor that activates individual var genes in the wild-type parasite still remains unknown. It may be a mechanism that randomly turns on var genes at a low rate. We previously found that only 1 in 200 parasites expresses the reticulocyte binding protein-like homologue 4 (Rh4) ligand in Dd2 (ref. 26), controlled by H3K9me3 (ref. 27), and a similar mechanism involving PfSETvs may exist for var genes. Recent work demonstrates that PfEMP1 proteins are key targets of humoral immunity ${ }^{28}$. However, malaria immunity is acquired only slowly after years of repeated exposure that, in part, reflects the time required for an individual to experience a sufficient number of variant antigens. The SETvs $\Delta$ parasite could be used as an antimalarial vaccine because of its ability to express all PfEMP1 proteins, to which the antibody would provide efficient protective immunity against malaria.

\section{METHODS SUMMARY}

Gene knockout in P. falciparum clones 3D7 and Dd2 was carried out using the double-crossover recombination strategy. After PCR screening, the positive knockout parasites were cloned and confirmed by Southern blot analyses. Transcriptome changes in each 3D7 knockout clone were initially analysed by the PFSANGER Affymetrix array at indicated time points after invasion. Transcriptional upregulation of most of var genes in either 3D7SETvs $\Delta$ or Dd2SETvs $\Delta$ at $18 \mathrm{~h}$ after invasion were further corroborated by qPCR. To evaluate the co-expression of multiple var genes in individual iRBCs, two-colour RNA FISH using different var gene probes and live cell IFA with rat and rabbit antibodies to different PfEMP1 proteins were performed at $18 \mathrm{~h}$ after invasion of 3D7SETvs $\Delta$. Our phylogenetic analysis (Supplementary Fig. 5) strongly suggested PfSETvs as a H3K36 methyltransferase. We therefore investigated the distribution changes at global level of H3K36me2/3 in 3D7 caused by PfSETvs $\Delta$ by ChIP-seq assay. As controls, we tested other histone methylations (H3K4me3, H3K9me3 and H4K20me3) in parallel. In addition, histone modification changes at the TSS region of var genes were investigated by ChIPqPCR. To explore the biological function of PfSETvs in regulating var gene silencing further, a triple HA tag was fused in frame to the $\mathrm{C}$ terminus of PfSETvs in 3D7SETvsHA by allelic exchange as described previously ${ }^{11}$. For strand-specific qPCR with reverse transcription assay, transcription of antisense lncRNAs driven by the var intronic promoter was investigated at five indicated time points after invasion of 3D7SETvs $\Delta$ or wild-type 3D7. DNA primers used in this study are listed in Supplementary Table 11

Full Methods and any associated references are available in the online version of the paper.

Received 19 October 2012; accepted 7 June 2013.

Published online 3 July 2013.

1. Miller, L. H., Baruch, D. I., Marsh, K. \& Doumbo, O. K. The pathogenic basis of malaria. Nature 415, 673-679 (2002)

2. Scherf, A., Lopez-Rubio, J. J. \& Riviere, L. Antigenic variation in Plasmodium falciparum. Annu. Rev. Microbiol. 62, 445-470 (2008).

3. Deitsch, K. W., Lukehart, S. A. \& Stringer, J. R. Common strategies for antigenic variation by bacterial, fungal and protozoan pathogens. Nature Rev. Microbiol. 7, 493-503 (2009).

4. Chookajorn, T. etal. Epigenetic memory at malaria virulence genes. Proc. Nat/ Acad. Sci. USA 104, 899-902 (2007)

5. Lopez-Rubio, J. J. etal. $5^{\prime}$ flanking region of var genes nucleate histone modification patterns linked to phenotypic inheritance of virulence traits in malaria parasites. Mol. Microbiol. 66, 1296-1305 (2007)

6. Lopez-Rubio, J. J., Mancio-Silva, L. \& Scherf, A. Genome-wide analysis of heterochromatin associates clonally variant gene regulation with perinuclear repressive centers in malaria parasites. Cell Host Microbe 5, 179-190 (2009).

7. Salcedo-Amaya, A. M. et al. Dynamic histone $\mathrm{H} 3$ epigenome marking during the intraerythrocytic cycle of Plasmodium falciparum. Proc. Natl Acad. Sci. USA 106, 9655-9660 (2009)

8. Tonkin, C. J. et al. Sir2 paralogues cooperate to regulate virulence genes and antigenic variation in Plasmodium falciparum. PLoS Biol. 7, e84 (2009).

9. Duraisingh, M. T. etal. Heterochromatin silencing and locus repositioning linked to regulation of virulence genes in Plasmodium falciparum. Cell 121, 13-24 (2005).

10. Cui, L., Fan, Q. \& Miao, J. Histone lysine methyltransferases and demethylases in Plasmodium falciparum. Int. J. Parasitol. 38, 1083-1097 (2008).
11. Volz, J. C. et al. PfSET10, a Plasmodium falciparum methyltransferase, maintains the active var gene in a poised state during parasite division. Cell Host Microbe 11 7-18 (2012).

12. Aravind, L., Abhiman, S. \& lyer, L. M. Natural history of the eukaryotic chromatin protein methylation system. Prog. Mol. Biol. Transl. Sci. 101, 105-176 (2011).

13. Joergensen, L. et al. Surface co-expression of two different PfEMP1 antigens on single Plasmodium falciparum-infected erythrocytes facilitates binding to ICAM1 and PECAM1. PLoS Pathog. 6, e1001083 (2010)

14. Newbold, C. I., Pinches, R., Roberts, D. J. \& Marsh, K. Plasmodium falciparum: the human agglutinating antibody response to the infected red cell surface is predominantly variant specific. Exp. Parasitol. 75, 281-292 (1992).

15. Carrozza, M. J. et al. Histone $\mathrm{H} 3$ methylation by Set2 directs deacetylation of coding regions by Rpd3S to suppress spurious intragenic transcription. Cell $\mathbf{1 2 3}$ 581-592 (2005).

16. Kizer, K. O. et al. A novel domain in Set2 mediates RNA polymerase II interaction and couples histone H3 K36 methylation with transcript elongation. Mol. Cell. Biol. 25, 3305-3316 (2005)

17. Barski, A. et al. High-resolution profiling of histone methylations in the human genome. Cell 129, 823-837 (2007).

18. Epp, C., Li, F., Howitt, C. A. Chookajorn, T. \& Deitsch, K. W. Chromatin associated sense and antisense noncoding RNAs are transcribed from the var gene family of virulence genes of the malaria parasite Plasmodium falciparum. RNA 15, 116-127 (2009).

19. Rechtsteiner, A. et al. The histone H3K36 methyltransferase MES-4 acts epigenetically to transmit the memory of germline gene expression to progeny. PLoS Genet 6, e1001091 (2010).

20. Tanaka, Y. et al. Dual function of histone H3 lysine 36 methyltransferase ASH1 in regulation of Hox gene expression. PLOS ONE 6, e28171 (2011).

21. Wu, S. F., Zhang, H. \& Cairns, B. R. Genes for embryo development are packaged in blocks of multivalent chromatin in zebrafish sperm. Genome Res. 21, 578-589 (2011).

22. Chantalat, S. et al. Histone $\mathrm{H} 3$ trimethylation at lysine 36 is associated with constitutive and facultative heterochromatin. Genome Res. 21, 1426-1437 (2011).

23. Venkatesh, S. et al. Set2 methylation of histone $H 3$ lysine 36 suppresses histone exchange on transcribed genes. Nature 489, 452-455 (2012).

24. Kim, T., Xu, Z., Clauder-Munster, S., Steinmetz, L. M. \& Buratowski, S. Set3 HDAC mediates effects of overlapping noncoding transcription on gene induction kinetics. Cell 150, 1158-1169 (2012).

25. van Werven, F. J. et al. Transcription of two long noncoding RNAs mediates matingtype control of gametogenesis in budding yeast. Cell 150, 1170-1181 (2012)

26. Soubes, S. C., Wellems, T. E. \& Miller, L. H. Plasmodium falciparum: a high proportion of parasites from a population of the Dd2 strain are able to invade erythrocytes by an alternative pathway. Exp. Parasitol. 86, 79-83 (1997).

27. Jiang, L. et al. Epigenetic control of the variable expression of a Plasmodium falciparum receptor protein for erythrocyte invasion. Proc. Natl Acad. Sci. USA 107, 2224-2229 (2010)

28. Chan, J. A. et al. Targets of antibodies against Plasmodium falciparum-infected erythrocytes in malaria immunity. J. Clin. Invest. 122, 3227-3238 (2012).

Supplementary Information is available in the online version of the paper.

Acknowledgements We thank S. K. Pierce at the National Institute of Allergy and Infectious Diseases, National Institutes of Health, for critical suggestions on the manuscript and A.F. Cowman for providing the pHTK transfection plasmid. We also thank V. Nair and E. Fisher at the NIH Research Technology Branch for assistance with electron microscopy. This research was supported by the Intramural Research Program of the National Institute of Allergy and Infectious Disease and the National Heart, Lung, and Blood Institute, National Institutes of Health, and also by the Nationa Natural Science Foundation of China (81271863) and the Key Research Program of the Chinese Academy of Sciences (KSZD-EW-Z-003-1-2). A.S. was supported by an ERC grant PlasmoEscape (250320). All authors have reviewed and agreed with the content of the manuscript.

Author Contributions L.J. and L.H.M. conceived and designed experiments. L.J. and J.M. performed the majority of the experiments. L.J., Q.Z. and A.S. performed FISH assays and analysed the data. L.J. and P.S. performed IFA and EM assays. L.J., J.M., T.N., W.Y.,W.P.,Y.W.,Y.L. J.Z and K.Z. performed ChIP-seq assays and analysed the data. L.J., J.M. and J.M.C.R. performed microarray analysis. K.R., L.T., T.L., T.G.T., A.B., G.W. and Q.J. generated reagents. L.A. performed phylogenetic analyses. L.J., J.M. and L.H.M. analysed all the data and wrote the manuscript. All authors discussed and edited the manuscript.

Author Information Microarray raw data have been deposited in MIAME format into the Gene Expression Omnibus (GEO) database under accession number GSE47349, and ChIP-seq raw data have been deposited into the Sequence Read Archive (SRA) database under accession number SRP022761. Reprints and permissions information is available at www.nature.com/reprints. The authors declare no competing financial interests. Readers are welcome to comment on the online version of the paper. Correspondence and requests for materials should be addressed to L.J. (Ibjiang@ips.ac.cn) or L.H.M. (Imiller@niaid.nih.gov). 


\section{METHODS}

Parasite culture and transfection. P. falciparum clones 3D7 (initially isolated from the Netherland $\mathrm{s}^{29}$ ) and Dd2 (initially isolated from Vietnam ${ }^{30}$ ) were cultured in human $\mathrm{O}^{+}$erythrocytes according to standard procedures ${ }^{31}$. For gene deletion, PCR amplification was performed on P. falciparum strain 3D7 genomic DNA to obtain gene-specific $5^{\prime}$ and $3^{\prime}$ flanking fragments, which were cloned into Spe I/ BglII (5')- and EcoR I/NcoI ( $\left.3^{\prime}\right)$-digested pHTK vector ${ }^{32}$. Names of the twelve targeted genes (Fig. 1d) and PCR primers are listed in Supplementary Table 11. Transfection and knockout selection were performed as described previously $y^{32}$. In brief, $250 \mu$ of packed iRBCs (5-10\% ring parasites) were transfected by electroporation with $100 \mu \mathrm{g}$ of the transfection pHTK plasmid. Positive (WR99210, $2 \mathrm{nM}$ ) and negative (ganciclovir, $20 \mu \mathrm{M}$ ) drug selection were applied for selecting a population of parasites in which the plasmid-derived human DHFR gene (for WR99210 selection) had been integrated via double crossover homologous recombination into the endogenous targeted gene locus, and the episomal plasmid carrying the Herpes simplex virus 2 TK gene (for ganciclovir self-killing selection). Selected knockout parasites were further confirmed by PCR screening (See also Supplementary Fig. 1a) before being cloned by limiting dilution.

Antibody. A peptide (CNTKAFKSKKLKLRK) from the PfSETvs protein was synthesized, and rabbits were immunized to obtain the polyclonal antibody to PfSETvs by GenScript. Various PfEMP1 domains (See also Supplementary Fig. 3b) were recombinantly expressed in a baculovirus system and immunized to rats and rabbits for making polyclonal antibodies to different PfEMP1 proteins, as described previously ${ }^{13}$.

Southern blotting. Southern blot analyses on PfSETvs $\Delta$ or PfSETvsHA parasites were performed using the DIG High Prime DNA Labelling and Detection Starter Kit (Roche) according to the product manual. In brief, genomic DNA was digested by EcoRV for $4 \mathrm{~h}$ at $37^{\circ} \mathrm{C}$ and separated on a $0.8 \%$ agarose gel for Southern blotting onto the Hybond $\mathrm{N}^{+}$nylon transfer membrane (Amersham). The target genomic DNA bands were hybridized by a digoxigenin-labelled DNA probe complementary to the homologous $3^{\prime}$ flanking fragment (See P in Fig. 1a) and detected by anti-digoxigenin-alkaline phosphatase conjugated antibody. Primers for the amplification of the probe are listed in Supplementary Table 11.

Western blotting. To determine knockout of PfSETvs at the protein level in 3D7SETvs $\Delta$, total parasite proteins extracted at $18 \mathrm{~h}$ after invasion were separated on $4-12 \%$ NuPAGE denature gel (Life Technologies) for western blot analysis using the rabbit antisera to the PfSETvs peptide and detected by an enhanced chemiluminescence (ECL) kit (Thermo Scientific). Total proteins from wild-type 3D7 were analysed as a control. Anti-PfSETvs peptide, diluted at a ratio of 1:300, and the secondary horseradish peroxidase-conjugated goat anti-rabbit IgG (Sigma), diluted 1:10,000, were incubated with western blot polyvinylidene difluoride (PVDF) membrane for ECL development. To determine reaction specificity of a commercial antibody to $P$. falciparum H3K36me3 (Cell Signaling), $1 \mu \mathrm{g}$ each of four synthesized peptides with the $P$. falciparum-specific histone H3K36 sequence (PfH3K36, biotin-GIKKPHRYRPG; PfH3K36me1, biotin-GIK(me)KPHRYRPG; PfH3K36me2, biotin-GIK(me2)KPHRYRPG; PfH3K36me3, biotin-GIK(me3) KPHRYRPG) was dotted on the PVDF membrane for western blot analysis as described above. To detect the effect of PfSETvs $\Delta$ on histone lysine methylations, total parasite proteins from wild-type 3D7 and 3D7SETvs $\Delta$ extracted at $18 \mathrm{~h}$ and $42 \mathrm{~h}$ after invasion were carried out for western blot analysis using rabbit antibodies to H3K36me3 (Cell Signaling), H3K36me2 (Abcam), H3K4me3 (Abcam) and H3K9me3 (Millipore), respectively. Antibody to histone H3 (Millipore) was used as a control. Rabbit anti-HA (Abcam) was used to detect PfSETvs-HA in 3D7SETvSHA. The western blot analysis was performed as mentioned above.

Microarray analyses. To analyse global gene expression profiles in the asexual stage, RNA from wild-type 3D7 and 3D7SETvs $\Delta$ were extracted from highly synchronized parasite cultures at $18 \mathrm{~h}$ (ring), $30 \mathrm{~h}$ (trophozoite) and $42 \mathrm{~h}$ (schizont) after invasion by using TRIzol (Life Technologies) according to the product manual and further digested with RNase free DNase (Ambion) to remove the DNA contamination. RNA hybridization was performed using the PFSANGER Affymetrix array at the microarray facility of the National Cancer Institute. PFSANGER Affymetrix arrays are high-density $8-\mu \mathrm{m}$ custom 25 -mer oligonucleotide arrays, whose tiling-like design was based on the $P$. falciparum (3D7) genome. In brief, $10 \mu \mathrm{g}$ of total RNA was reverse-transcribed and biotin-labelled. Hybridizations were carried out at $45^{\circ} \mathrm{C}$ for $16 \mathrm{~h}$ with constant rotation at $60 \mathrm{~g}$. Gene arrays were then scanned at an emission wavelength of $570 \mathrm{~nm}$ at $1.56 \mu \mathrm{m}$ pixel resolution using a confocal scanner (Affymetrix GeneChip Scanner 3000 $7 \mathrm{G})$. After scanning, the hybridization intensity for each 25 -mer feature was computed using Affymetrix GCOS version 1.3 software $^{33}$. The raw data was then transferred to our in-house software for background adjustment, normalization and summarization of the probe sets.

qPCR. For qPCR analysis, RNA was isolated and purified as described above. First, strand complementary DNA was synthesized by either random primer mixes or gene-specific primers using Superscript III Reverse Transcriptase (Life Technologies) according to product manual. PCR primers used for detecting mRNA expression of 3D7 var genes were as described previously ${ }^{34}$. Primers for detecting transcripts from each Dd2 var gene and for 3D7 var lncRNAs were designed in this study (Supplementary Table 11). qPCR was performed on a iQ5 Multi-colour Realtime PCR Detection System (Bio-Rad) with a program of 1 cycle of 5 min at $95^{\circ} \mathrm{C} ; 40$ cycles of $30 \mathrm{~s}$ at $95^{\circ} \mathrm{C}, 30 \mathrm{~s}$ at $50^{\circ} \mathrm{C}$ and $60 \mathrm{~s}$ at $60^{\circ} \mathrm{C}$. A housekeeping gene, arginyltRNA synthetase (PF3D7_0913900), was used to normalize the transcriptional level of each var gene.

Live-cell-infected RBC IFA. Live-cell IFA for infected RBCs was performed as described previously with minor modifications ${ }^{13}$. In brief, iRBCs were washed in $1 \%$ BSA in PBS (BSA/PBS) and the pellet was re-suspended in $200 \mu \mathrm{l} \mathrm{BSA/PBS}$ Antibodies specific for various PfEMP1 proteins listed in Supplementary Fig. 3b were used at a 1:50 dilution and incubated at room temperature $\left(23^{\circ} \mathrm{C}\right)$ for $30 \mathrm{~min}$ After washing three times in BSA/PBS, cells were fixed with $2.5 \%$ paraformaldehyde and $0.01 \%$ glutaraldehyde for $10 \mathrm{~min}$ at room temperature and washed with BSA/PBS. Subsequently, cells were incubated with Alexa 488-conjugated goat anti-rabbit IgG (Life Technologies) and Alexa 594-conjugated goat anti-rat IgG (Life Technologies) for $30 \mathrm{~min}$ at room temperature and washed with BSA/PBS containing $0.1 \%$ Triton X-100 and mounted with prolong gold DAPI. Images were captured on a Leica SP2 confocal microscope and visualized using Bitplane Imaris software.

Scanning and transmission electron microscopy. Scanning and transmission electron microscopy were performed as described previously with modifications $s^{35,36}$ For scanning electron microscopy (SEM), iRBCs were gently allowed to settle on silicon chips for $20 \mathrm{~min}$ at room temperature in an 8-well chamber slide (Labtek). Freshly prepared fixative (2.5\% glutaraldehyde, $3 \%$ paraformaldehyde, $0.05 \mathrm{M}$ phosphate buffer, $4 \%$ sucrose) was added to the cells and incubated at room temperature for $1 \mathrm{~h}$. All subsequent processing was carried out in a Pelco Biowave laboratory microwave system (Ted Pella) at $250 \mathrm{~W}$ and 20 in $\mathrm{Hg}$ (mercury) vacuum. The chips were post-fixed with $1 \%$ osmium tetroxide- $0.8 \%$ potassium ferricyanide in $0.1 \mathrm{M}$ sodium cacodylate, followed by rinsing with water and dehydration in a graded ethanol series. The specimen was critical point dried in a Bal-Tec CPD 030 drier (Bal-Tec AG) and coated with $80 \AA$ of iridium using an IBS ion beam sputter (South Bay Technology). SEM samples were imaged using a Hitachi SU8000 SEM (Hitachi High Technologies). For transmission electron microscopy, parasites were fixed with $2.5 \%$ glutaraldehyde, $3 \%$ paraformaldehyde, $0.05 \mathrm{M}$ phosphate buffer and $4 \%$ sucrose at room temperature for $2 \mathrm{~h}$. The cells were post-fixed in a microwave with $1 \%$ osmium tetroxide- $0.8 \%$ potassium ferricyanide in $0.1 \mathrm{M}$ sodium cacodylate, followed by $1 \%$ tannic acid in distilled water, and stained en bloc with $1 \%$ aqueous uranyl acetate. They were then rinsed with distilled water and dehydrated in a graded ethanol series. The pellets were then infiltrated and embedded in Spurr's resin which was polymerized overnight in a $68^{\circ} \mathrm{C}$ oven. Thin sections $(90 \mathrm{~nm})$ were cut using a UC6 ultramicrotome (Leica Microsystems) and stained with $4 \%$ aqueous uranyl acetate and Reynold's lead citrate before viewing on a $120 \mathrm{kV}$ Tecnai Biotwin Spirit TEM (FEI). Digital images were acquired with a Hamamatsu XR-100 digital camera system.

FISH. Synchronized ring-stage parasites were released from iRBCs by $0.15 \%$ saponin treatment followed by fixation with $4 \%$ paraformaldehyde in $1 \times \mathrm{PBS}$ overnight at $4{ }^{\circ} \mathrm{C}$. The fixed parasites were washed twice with $1 \times \mathrm{PBS}$, then deposited on a microscope slide (Fisher Scientific) as a monolayer and subjected to RNA FISH in the conditions as described previously ${ }^{6}$. For combined immunoRNA FISH, parasites were deposited on slides and treated with $0.1 \%$ Trition X-100 in $1 \times$ PBS for $5 \mathrm{~min}$ before hybridization of RNA FISH. After incubation of parasites with FISH probe at $42{ }^{\circ} \mathrm{C}$ for $16 \mathrm{~h}$, the slides were washed three times with $2 \times$ saline-sodium citrate buffer and fixed again in $4 \%$ paraformaldehyde for $15 \mathrm{~min}$ before IFA for detection of H3K36me3 by using the antibody to H3K36Me3 (Cell Signaling) with 1:100 dilution. For the individual var genespecific RNA FISH probes, DNA templates were amplified by PCR from 3D7 genomic DNA with primers shown in Supplementary Table 11. For the template of the exon 2 probe for the var gene family, the exon 2 regions were amplified with types A, B and C primer sets as described previously ${ }^{6}$. The products were pooled for labelling. The PCR products were purified by Gel Extraction kit (Qiagen) and used in probe preparation with a Biotin- or a Fluorescein-High Prime kit (Roche). Images were captured by using a Nikon Eclipse 80i microscope with a CoolSnap HQ2 camera (Photometrics). Primers used in amplification of individual var probes are described in Supplementary Table 11.

ChIP-seq and ChIP-qPCR. Highly synchronous cultures of ring-, trophozoiteand schizont-stage parasites were used for the ChIP study. Crosslinked chromatin was prepared by adding $1 \%$ formaldehyde to the culture for $5 \mathrm{~min}$ followed by addition of glycine to $0.125 \mathrm{M}$ final concentration. After saponin lysis, nuclei were isolated by homogenization in $10 \mathrm{mM}$ Tris at $\mathrm{pH} 8.0,3.0 \mathrm{mM} \mathrm{MgCl} 2$ and $0.2 \%$ Nonidet P-40, and collected on a $0.25 \mathrm{M}$ sucrose-buffer cushion and suspended in 
SDS buffer (1\% SDS, $50 \mathrm{mM}$ Tris, pH 8.0, $10 \mathrm{mM}$ EDTA, protease inhibitors). Chromatin was sheared by sonication in a Bioruptor UCD-200 (Diagenode) for $10 \mathrm{~min}$ at 30 -s intervals, power setting high, to a size of $300-800 \mathrm{bp}$. Chromatin samples were frozen and stored at $-80^{\circ} \mathrm{C}$. ChIP was performed as described previously ${ }^{17}$. In brief, commercially available antibodies to H3K36me3 (Cell Signaling), H3K4me3 (Abcam), H3K9me3 (Millipore), H3K20me3 (Abcam) and histone $\mathrm{H} 4 \mathrm{~K} 5 / \mathrm{K} 8 / \mathrm{K} 12 / \mathrm{K} 16$ acetylation (Abcam) were added to crosslinked samples of wild-type 3D7 and 3D7SETvs $\Delta$, or a mouse anti-HA (Abcam) to 3D7SETvsHA samples, and incubated at $4{ }^{\circ} \mathrm{C}$, followed by the addition of $10 \mu \mathrm{l}$ $\mathrm{A} / \mathrm{G}$ beads and further incubation for $2 \mathrm{~h}$. After washing with buffers containing 100,150 and $250 \mathrm{mM} \mathrm{NaCl}$, immuno-precipitated DNA was eluted and purified using PCR purification columns (Qiagen). The resulting double-stranded DNA was then end repaired, followed by adding an A base at the ends. Illumina pairedend index adaptor was ligated and size selected. A 16-cycle PCR was then carried out with Phusion Hot Start High-Fidelity DNA Polymerase (Finnzymes) to generate the final ChIP-seq library. We used Illumina HiSeq 2000 to perform the single-end sequencing ( 50 cycles). Quality sequencing reads were mapped against the Plasmodium falciparum genome assembly (PlasmoDB v8.2) with BurrowsWheeler Alignment tool (BWA) using default parameters. ChIP-qPCR was performed for different gene regions (TSS, $3^{\prime}$ end of exon 1) as well as antisense transcription level in a iQ5 Multi-colour Real-time PCR Dection System (BioRad) using primer sets described in Supplementary Table 11.

Tree construction and topology testing. Sequences of the SETD2-NSD-ASH1 clade to span a comprehensive phyletic range across eukaryotes were collected using the Position-Specific Iterative Basic Local Alignment Search Tool (PSI-BLAST) program. The SET domains and the associated AWS domains were aligned using the MUSCLE program. The tree was constructed using two methods: (1) a preliminary tree was obtained using the approximately-maximum-likelihood method implemented in the FastTree 2.1 program under default parameters. This gave an idea of the positions of key members; and (2) a complete tree was constructed using the MEGA 5.1 program with the following parameters: four distinct gamma distributed rate categories and one invariant were used for modelling among site variation, the WAG matrix with frequencies, was used as the substitution model; the maximum-likelihood searched used the close neighbour exchange method. The tree was bootstrapped using 10,000 resampling of estimated log-likelihood bootstrap percentage resamplings with the MOLPHY package. The tests for alternative topology were carried out using the CONSEL program for the ShimodairaHasegawa test and these overwhelmingly rejected the grouping of the apicomplexan clade with either the NSD subclade $\left(P<10^{-4}\right)$ or the SETD2 subclade $\left(P<10^{-7}\right)$

29. Walliker, D. et al. Genetic analysis of the human malaria parasite Plasmodium falciparum. Science 236, 1661-1666 (1987).

30. Wellems, T. E. et al. Chromosome size variation occurs in cloned Plasmodium falciparum on in vitro cultivation. Rev. Bras. Genet. 11, 813-825 (1988).

31. Trager, W. \& Jensen, J. B. Human malaria parasites in continuous culture. Science 193, 673-675 (1976)

32. Duraisingh, M. T., Triglia, T. \& Cowman, A. F. Negative selection of Plasmodium falciparum reveals targeted gene deletion by double crossover recombination. Int. J. Parasitol. 32, 81-89 (2002)

33. Jiang, H. etal. High recombination rates and hotspots in a Plasmodium falciparum genetic cross. Genome Biol. 12, R33 (2011)

34. Salanti, A. et al. Selective upregulation of a single distinctly structured var gene in chondroitin sulphate A-adhering Plasmodium falciparum involved in pregnancyassociated malaria. Mol. Microbiol. 49, 179-191 (2003).

35. Srinivasan, P. et al. Binding of Plasmodium merozoite proteins RON2 and AMA1 triggers commitment to invasion. Proc. Natl Acad. Sci. USA 108, 13275-13280 (2011).

36. Tahlan, K. et al. SQ109 targets MmpL3, a membrane transporter of trehalose monomycolate involved in mycolic acid donation to the cell wall core of Mycobacterium tuberculosis. Antimicrob. Agents Chemother. 56, 1797-1809 (2012). 